\title{
Téoros
}

Revue de recherche en tourisme

\section{Les clientèles touristiques : un tour d'horizon}

\section{Marcel Samson}

Volume 4, numéro 2, juillet 1985

Les clientèles touristiques : un tour d'horizon

URI : https://id.erudit.org/iderudit/1080623ar

DOI : https://doi.org/10.7202/1080623ar

Aller au sommaire du numéro

Éditeur(s)

Université du Québec à Montréal

ISSN

0712-8657 (imprimé)

1923-2705 (numérique)

Découvrir la revue

Citer ce document

Samson, M. (1985). Les clientèles touristiques : un tour d'horizon. Téoros, 4(2),

1-1. https://doi.org/10.7202/1080623ar d'utilisation que vous pouvez consulter en ligne.

https://apropos.erudit.org/fr/usagers/politique-dutilisation/ 


\section{Présentation}

\section{Les clientèles touristiques: un tour d'horizon}

Dans ce numéro de la revue Téoros sur les clientèles touristiques, nous avons voulu établir une sorte de bilan en confrontant les contributions de différentes personnesressources sur le sujet.

Nous avons abordé ce theme de trois facons: d'abord, une analyse de la situation présente et passée de la clientèle touristique, nous présentons ensuite le profil vacancier de trois catégories de population; enfin, nous conchons sur des nouvelles pratiques et des perspectives d'avenir.

Tenter une description de l'évolution de la clientèle touristique du Québec durant les vingt dernières années n'est pas une mince tâche. L'obstacle est de taille, les séries statistiques font cruellement defaut. Notre collaborateur Jacques Demers a pu y compenser en faisant appelà sa bonne connaissance de l'évolution du phénomène touristique au Québec. Cependant, cette carence illustre bien que l'étude de cette pratique de consommation est encore récente au Québec. // y a une quinzaine d'années à peine, on aurait été incapable d'avancer de chiffres précis; aujourd'hui, on commence à y voir beaucoup plus clair. Ainsi, les documents de la Direction Analyse et Développement du ministère du Tourisme sur I'état de la clientèle au Québec sont un apport important. Les différents concepts utilisés dans les enquêtes EVC (Enquêtes sur les voyages des Canadiens) que précise Patrick Cluzeau aideront le profane à s'y retrouver. On parle en effet icide voyages des Canadiens et les définitions des termes, plus précisément pour les voyages d'agrément, sont différentes de celles qu'utilise Marc Laplante pour faire l'analyse du comportement du vacancier québécois.

Cette dernière analyse, fort importante pour les intervenants touristiques régionaux, ouvre la porte à l'étude de trois segments de population dont le profil vacancier est relativement peu connu: les nouveaux retraités, les producteurs agricoles et la clientèle étudiante.
Dans I'avenir, on aurait tort de ne pas tenir compte des pratiques de consommation des nouveaux retraités. Les résultats de cette enquête dépassent nos prévisions les plus optimistes. D'autre part, l'étude de P. Filiatrault et $H$. Laroche sur les producteurs agricoles apportent un éclairage tout à fait nouveau sur les loisirs et le tourisme de cette clientèle rurale. Les données qu'ils ont obtenues inciteront, je pense, à poursuivre des recherches de ce côté. Nous avons aussi demandé à Luc Guertin, impliqué depuis quelques années dans le tourisme-jeunesse, de nous tracer un portrait de l'étudiant vacancier de 1985. Des propos qu'il nous tient, retenons que les nonvacanciers semblent nombreux parmi les jeunes. II faudrait sans doute des études plus complètes pour obtenir des données précises sur cette clientèle qui devrait sans doute explorer des voies nouvelles.

"Débanalisation" et entrepreneurship, voilà le prix qu'aura à payer l'industrie du tourisme au Québec pour ne pas régresser. Insuffler un dynamisme nouveau par l'innovation, c'est la perspective que nous devons choisir selon Jean-Marie Lucas-Girardville. Sinon, notre industrie touristique sera asphyxiée par la concurrence féroce de nos voisins. Des espoirs existent cependant. Selon André Hut, des initiatives socio-culturelles sont apparues qui ont des répercussions importantes sur les vacances et les loisirs de nos concitoyens.

$\dot{A}$ quoi ressemblera la clientèle touristique de demain? Elle aura certainement des comportements plus variés et surtout elle sera plus exigeante en termes de qualité de vie. Nous devons continuer à prendre son pouls pour en comprendre l'évolution et adapter notre équipement touristique.

\section{Marcel Samson}

